\title{
Evoked Potential Monitoring during Spine Surgery
}

\section{Siavash S Haghighi*}

Department of Neurodiagnostics, Sharp HealthCare, San Diego, California 92123, USA

\section{Editorial}

Intraoperative electrophysiological monitoring is used in a variety of procedures. Various techniques are thought to be useful for the detection and prevention of neurological deficits [1,2]. Mechanisms of thoraco-lumbar spinal cord injury during scoliosis surgery include ischemia and over-distraction of vertebral column. This injury can be potentially detected by standard tibial somatosensory evoked potentials (SSEPs) thus reducing the risk of permanent paraplegia [35]. However, SSEPs only detect sensory tracts dysfunction and motor deficits can occur without any SSEP changes [6,7]. Motor evoked potentials (MEP), obtained by transcranial cortical stimulation, can provide an early detection of motor disturbance [8-10]. The combined methods can improve the sensitivity and specificity of the monitoring [11].

\section{SSEPs (Somatosensory Evoked Potentials)}

Somatosensory evoked potentials (SSEPs) monitoring serves as an early warning system to detect injury intraoperatively. SSEP monitoring is performed to assess the functional status of peripheral nerves and sensory tracts in the spinal cord. SSEP is elicited by stimulating either a sensory or mixed peripheral nerve. The neuronal response to the electrical stimulation can be recorded from various locations along the neural pathway.

SSEPs can be significantly affected by the halogenated anesthetics. Inhalation anesthetics such as sevoflurane, desflurane, and isoflurane are most often used. All anesthetics affect cortical responses [12,13].

Intraoperative monitoring equipment consists of three systems: stimulus, recording, and data storage. The last component of the monitoring system is the signal averager. Signals that are time-locked to the presentation of the stimulus are converted to a digital signal. The computer captures a set of samples and computes the mean of each data point which is displayed on the monitor as a waveform.

\section{Neurogenic Motor Evoked Potentials (NMEPs)}

Stimulation is applied to the spinal cord and the descending sensory volley is recorded from the nerves in the extremities $[14,15]$.

\section{Transcranial Cortical Motor Evoked Potentials (TcMEP)}

Typically, a large current is required for adequate motor cortex stimulation. The resultant response can be recorded from spinal cord or target muscles. MEPs are extremely sensitive to inhalation anesthetics. Intravenous propofol/narcotic based anesthesia with or without nitrous oxide can provide favorable anesthesia to perform TcMEPs [16]. Currently, all patients with a history of epilepsy, skull fracture, craniotomy, cardiac pacemaker, or cochlear implant are excluded from the TcMEP use. Intraoperatively, since cortical stimulation, in non-paralyzed patient, can cause severe jaw musculature contraction, adequate measures should be taken to prevent tongue and teeth damage.

\section{Spinal Nerve Root Monitoring}

Pedicle screw instrumentation systems for spinal arthrodesis are in widespread use. Malpositioned screws can induce loss of fixation, neuronal injury, and pain syndromes. Intraoperative evoked EMG monitoring of pedicle screws has proven to be a simple, safe, and efficacious technique in accurate placement of pedicle screws [17]. A positive EMG response at or below the constant-current of $\leq 8 \mathrm{~mA}$ requires either inspection, redirection, or removal of the instrument or implant. Normal free- run EMG response is predictive of the lack of nerve root injury or irritation. An abnormal EMG response during a spine procedure may or may not be associated with a clinical injury.

\section{Dermatomal Sensory Evoked Potential Monitoring}

Dermatomal somatosensory evoked potentials (DSEP) are a more specific mean for nerve root monitoring than SSEP monitoring $[18,19]$. DSEP has been used to assess adequacy of root decompression. Data does not support the use of DSEP for improvement of outcome following lumbar decompression and fusion.

\section{Neuromonitoring During Decompression}

There is no medical evidence to support the hypothesis that intraoperative monitoring improves long term outcome following decompression procedures for degenerative diseases. Changes in SSEP/DSEP monitoring appear to be sensitive to nerve root injury during surgery. There is no tangible evidence to indicate that the use of monitoring provides useful information in assessing the adequacy of nerve root decompression.

\section{References}

1. Schmitt EW (1981) Neurological complications in the treatment of scoliosis. A sequential report of the Scoliosis Research Society 1971-1979.17th annual meeting of the Scoliosis Research Society.

2. Wilber RG, Thompson GH, Shaffer JW, Brown RH, Nash CL Jr (1984) Postoperative neurological deficits in segmental spinal instrumentation. A study using spinal cord monitoring. J Bone Joint Surg Am 66: 1178-1187.

3. Bieber E, Tolo V, Uematsu S (1988) Spinal cord monitoring during posterior spinal instrumentation and fusion. Clin Orthop Relat Res: 121-124.

4. Dinner DS, Lüders H, Lesser RP, Morris HH, Barnett G, et al. (1986) Intraoperative spinal somatosensory evoked potential monitoring. J Neurosurg 65: $807-814$

5. Friedman WA, Richards R (1988) Somatosensory evoked potential monitoring accurately predicts hemi-spinal cord damage: a case report. Neurosurgery 22 140-142.

6. Ginsburg HH, Shetter AG, Raudzens PA (1985) Postoperative paraplegia with preserved intraoperative somatosensory evoked potentials. Case report. J Neurosurg 63: 296-300.

7. Ben-David B, Haller G, Taylor P (1987) Anterior spinal fusion complicated by paraplegia: A case report of a false-negative somatosensory evoked potential. Spine (Phila Pa 1976) 12: 536-539.

*Corresponding author: Siavash S Haghighi, Department of Neurodiagnostics, Sharp HealthCare, San Diego, California 92123, USA, Tel: 858-939-3459; Fax 858-939-3466; E-mail: Siavash.haghighi@sharp.com

Received March 08, 2012; Accepted March 09, 2012; Published March 10, 2012

Citation: Haghighi SS (2012) Evoked Potential Monitoring during Spine Surgery. J Spine 1:e103. doi:10.4172/2165-7939.1000e103

Copyright: (c) 2012 Haghighi SS. This is an open-access article distributed under the terms of the Creative Commons Attribution License, which permits unrestricted use, distribution, and reproduction in any medium, provided the original author and source are credited. 
8. MacDonald DB, Al Zayed Z, Khoudeir I, Stigsby B (2003) Monitoring scoliosis surgery with combined multiple pulse transcranial electric motor and cortical somatosensory-evoked potentials from the lower and upper extremities. Spine (Phila Pa 1976) 28: 194-203.

9. Langeloo DD, Journée HL, Polak B, de Kleuver M (2001) A new application of TCE-MEP: spinal cord monitoring in patients with severe neuromuscular weakness undergoing corrective spine surgery. J Spinal Disord 14: 445-448.

10. Haghighi SS (2006) Transcranial stimulation parameters to elicit motor evoked potentials. Electromyogr Clin Neurophysiol 46: 409-412.

11. Burke D, Hicks R, Stephen J, Woodforth I, Crawford M (1992) Assessment of corticospinal and somatosensory conduction simultaneously during scoliosis surgery. Electroencephalogr Clin Neurophysiol 85: 388-396.

12. Sloan T, Sloan H, Rogers J (2010) Nitrous oxide and isoflurane are synergistic with respect to amplitude and latency effects on sensory evoked potentials. J Clin Monit Comput 24: 113-123.

13. Sloan TB, Heyer EJ (2002) Anesthesia for intraoperative neurophysiologic monitoring of the spinal cord. J Clin Neurophysiol 19: 430-443.

14. Su CF, Haghighi SS, Oro JJ, Gaines RW (1992) "Backfiring" in spinal cord monitoring. High thoracic spinal cord stimulation evokes sciatic response by antidromic sensory pathway conduction, not motor tract conduction. Spine (Phila Pa 1976) 17: 504-508.

15. Toleikis JR, Skelly JP, Carlvin AO, Burkus JK (2000) Spinally elicited peripheral nerve responses are sensory rather than motor. Clin Neurophysiol 111: 736742.

16. Calancie B, Klose KJ, Baier S, Green BA (1991) Isoflurane-induced attenuation of motor evoked potentials caused by electrical motor cortex stimulation during surgery. J Neurosurg 74: 897-904.

17. Glassman SD, Dimar JR, Puno RM, Johnson JR, Shields CB, et al. (1995) A prospective analysis of intraoperative electromyographic monitoring of pedicle screw placement with computed tomographic scan confirmation. Spine (Phila Pa 1976) 20: 1375-1379.

18. Kraft GH (2003) Dermatomal somatosensory-evoked potentials in the evaluation of lumbosacral spinal stenosis. Phys Med Rehabil Clin N Am 14: 71-75.

19. Storm SA, Kraft GH (2004) The clinical use of dermatomal somatosensory evoked potentials in lumbosacral spinal stenosis. Phys Med Rehabil Clin N Am 15: 107-115. 